\title{
Strates
}

STRATES Matériaux pour la recherche en sciences sociales

Hors-série | 2002

Parcours dans la recherche urbaine, Michel Rochefort, un géographe engagé

\section{Michel Rochefort et la Bourgogne}

\section{Françoise Plet}

\section{(2) OpenEdition}

Journals

Édition électronique

URL : http://journals.openedition.org/strates/551

DOI : $10.4000 /$ strates.551

ISSN : 1777-5442

Éditeur

Laboratoire Ladyss

Édition imprimée

Date de publication : 1 janvier 2002

ISSN : 0768-8067

Référence électronique

Françoise Plet, « Michel Rochefort et la Bourgogne », Strates [En ligne], Hors-série | 2002, mis en ligne le 19 mai 2005, consulté le 08 septembre 2020. URL : http://journals.openedition.org/strates/551 ;

DOI : https://doi.org/10.4000/strates.551

Ce document a été généré automatiquement le 8 septembre 2020

Tous droits réservés 


\title{
Michel Rochefort et la Bourgogne
}

\author{
Françoise Plet
}

1 Ou bien : un homme, ses lieux de jeunesse et ses premiers travaux. Car ce professeur dont le nom évoque la recherche sur "l'armature urbaine", la croissance et les structures sociales des grandes villes du Brésil, du Maghreb et d'Afrique, les politiques d'aménagement, cet habitant de Paris et citoyen de Sao Paulo, Brasilia, Recife, Tunis qu'il a tant fréquentées est aussi un rural profondément attaché à un terroir bourguignon, situé à une quinzaine de kilomètres au nord d'Avallon, qu'il continue d'habiter à ses heures de liberté. Comme son lointain collatéral l'écrivain Restif ${ }^{1}$ de la Bretonne de Sacy, à deux pas de son village natal de Joux-la-Ville, Michel Rochefort débarque à vingt ans à Paris et opère, comme lui, de fréquents retours sur ses terres. C'est cet attachement à des paysages familiers et familiaux que je voudrais restituer à travers des esquisses de territoires ruraux dont l'essentiel vient de Michel Rochefort lui-même ${ }^{2}$.

Au centre : Joux-la-Ville

2 C'est un gros village tassé au plus creux ${ }^{3}$ de l'une des vastes clairières agricoles des plateaux bourguignons de l'Yonne. Le paysage est formé de croupes uniformément rousses en été, sur lesquelles quelques tracteurs s'affairent à charger la paille, à déchaumer, ou à défricher; rousseur des chaumes et de l'argile de décalcification qui enrobe la caillasse superficielle, patiemment brisée par des générations paysannes. Mais en dépit de la puissance des outils actuels de concassage et d'extraction des blocs, cela reste une terre de pierres, trop peu profonde pour qu'y pousse autre chose que céréales et colza, et trop froide pour les cultures de printemps.

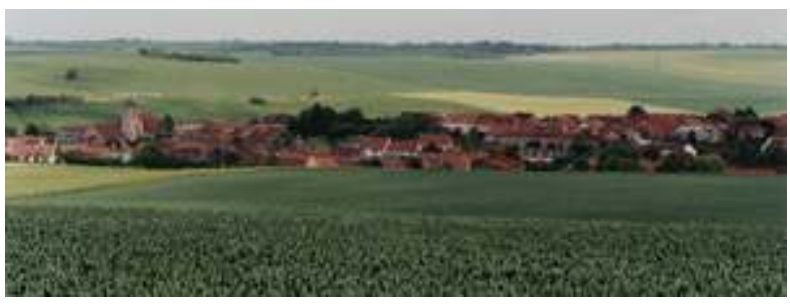

Le bourg de Joux. Photo Jean-Paul Rivaud, 1999.

3 De sorte que le vert y est couleur d'hiver dont les nuances seules impriment à l'œil le dessin des vastes parcelles de culture que ne ponctuent plus que quelques lanières de 
bois dont certaines sont bordées de cytises : c'était la passion de l'arrière-grand-père de Michel Rochefort, notaire à Joux, que d'en planter en bordure des parcelles dispersées qu'il rachetait et transformait en petits bois après que la crise du phylloxéra eut conduit à l'émigration une partie de la population, «parcelles qui n'étaient plus utilisées par personne et qu'il achetait pour une bouchée de pain». "Chaque fois que tu vois des cytises dans les petits bois qui restent, c'est une mémoire de l'arrière-grand-père ». Le jaune en effet anime les printemps de cette fin de siècle: les cytises hérités du passé parfois, et surtout le colza, la nouvelle grande culture.

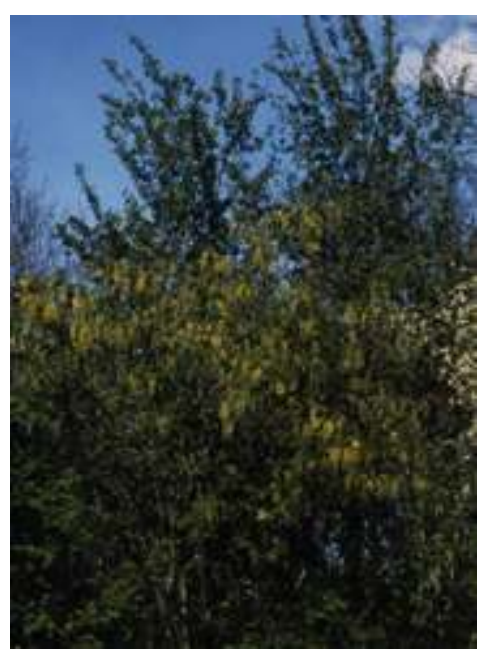

Cytises plantés par le grand-père, en bordure d'une barrière boisée. Photo François Plet, 1999.

4 La forêt marquait, marque encore les limites de la commune, même si elle a reculé face aux défrichements du dernier quart de siècle. Elle correspond aussi aux lieux les plus élevés, où la roche calcaire est au plus près de la surface, avec très peu ou pas du tout de sols.

5 À la périphérie, des fermes isolées. Des hameaux, invisibles du bourg, se nichent dans des vallons enserrés par l'herbe et par un maquis de prunelliers et d'aubépine qui tapisse leurs pentes raides entre des alignements de murgers ${ }^{4}$ plus ou moins imposants, plus ou moins réutilisés par les bricoleurs ou pour recharger les chemins.

6 C'est au cœur de ce paysage un peu austère que se situe la maison natale, grande bâtisse sur cour au cœur du bourg, ancienne maison de gros vigneron et marchand de vins achetée en 1896 par le notaire de Joux pour en faire la résidence du jeune ménage constitué par l'une de ses filles et le nouveau médecin du village. L'ancien cabinet médical à droite de la cour charretière, à gauche la grange et l'écurie pour les chevaux en arrière de laquelle, côté jardin, logeaient les domestiques, au centre l'habitation. Une petite aile sur jardin a été construite pour loger les deux filles du médecin. Ce médecin, mort en 1939, était le grand-père de Michel Rochefort. La maison fut son lieu de naissance, le lieu de vacances de ses parents, le lieu de ses étés de jeunesse. Elle est aujourd'hui sa retraite des périodes de repos, d'intimité, ou d'amitié.

L'histoire d'une famille dans l'histoire d'un village : de la bourgeoisie rurale à l'école de la République 


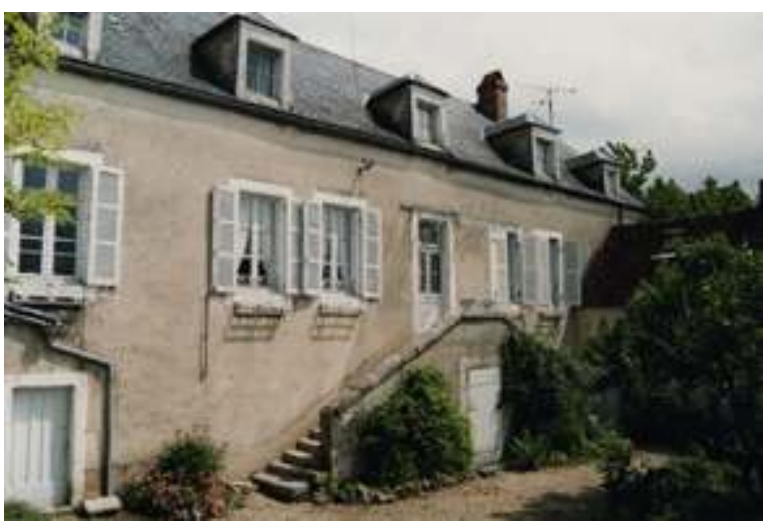

La maison natale. Photo Jean-Paul Rivaud, 1999.

Village et famille se mêlent étroitement et la connaissance de l'un et de l'autre se construisent mutuellement dans les pratiques et dans la mémoire de Michel Rochefort. Michel Rochefort est né en 1927 de la fille du médecin et du fils de l'instituteur de Joux.

La branche maternelle de sa famille se rattache à une bourgeoisie rurale très localisée, parente des Restif de Sacy. Marie-Anne Rétif, demi-sœur de l'écrivain ${ }^{5}$ épousa un tonnelier de Joux et Nicolas Restif de la Bretonne est allé à l'école à Joux, logeant chez sa sœur, avant d'apprendre le métier d'imprimeur à Auxerre. C'est cette branche, cette filiation qui sont valorisées et reconnues dans la famille étendue dont fait partie Jean Chamant, sénateur de l'Yonne, qui fut ministre, président du conseil général de l'Yonne, conseiller général de Quarré-les-Tombes et maire d'Avallon.

Les activités et l'origine de la fortune de la branche Rétif de la famille ne sont, selon Michel Rochefort, pas claires au XVIII ${ }^{e}$ siècle. Sans doute étaient-ils régisseurs des biens du seigneur, l'abbaye de Régny dans la vallée de la Cure. Ils résidaient à Massangis, à dix kilomètres à l'est de Joux, et achetaient des terres. Au premier cadastre, ici de 1839, ils possédaient 150 hectares au hameau de Oudun, hameau de défrichement monastique, soit deux fermes et 50 hectares de forêt issus des biens nationaux, et une ferme au hameau de Puits-d'Edme.

11 Dans La vie de mon père ${ }^{6}$, Edme Restif de la Bretonne, né en 1692 à Nitry, autre commune limitrophe de Joux, migre à Sacy par mariage et s'y occupe d'abord des terres de son beau-père, puis y devient défricheur en créant en 1713 un grand domaine personnel à l'écart du village ; il l'arrondit en achetant tout ce qui était à vendre, et lui applique les principes des physiocrates. Il devint un notable reconnu, officier de justice, alors que "jusque là [...] les bons officiers de la justice [...] étaient de Vermenton ", dont la juridiction, à sa mort, comprenait aussi Vermenton. En 1729, il devient également «administrateur pour les trois seigneurs ».

12 Le premier des ancêtres directs installé à Joux, dont Michel Rochefort ait connaissance, était un officier de santé des armées napoléoniennes, rescapé de la retraite de Russie, fils des Rétif de Massangis. Son fils fut médecin à Joux, son petit-fils, notaire dans la même commune, maire et conseiller d'arrondissement.

13 La phase notaire de l'histoire familiale est mal connue, sorte de symbole d'échec et de ruine, de ce que Michel Rochefort appelle la chute de la bourgeoisie rurale dans la première moitié du siècle. Outre la grande ferme d'Oudun, son arrière-grand-père le notaire possédait deux hectares de vignoble dans un hameau aujourd'hui détruit, 
Couchenoire, des fermes et des bois. Mais il était en 1900 en difficulté financière, en raison de la crise agricole qui a culminé ici en 1902. Les fermes ne valaient plus rien. Il est mort à ce moment là, à 52 ans, victime d'un accident de chasse, en laissant de nombreuses dettes. Une partie de ses biens fut alors vendue, dont sa maison avec parc devenue depuis la mairie du village. Il ne restait plus du domaine qu'Oudun et quelques bois que rachète le gendre médecin.

C'est en 1896, alors qu'il n'y avait plus de médecin au village, que ce jeune médecin issu d'une famille paysanne enrichie sous la Révolution, dont le père était aussi médecin à Voutenay-sur-Cure, s'était installé à Joux. Le docteur Lecourt y avait épousé la fille aînée du notaire, âgée de 16 ans. En 1928, un ami banquier lui conseilla la vente de la ferme qui lui restait et le placement du produit en actions. Las! en 1932, ces actions n'étaient plus que papier. Le grand-père médecin meurt en 1939. La grand-mère vend ce qui lui reste au fur et à mesure des besoins d'un quotidien difficile ${ }^{7}$, ruinée en deux crises majeures : la crise du phylloxéra suivie de celle des matières premières agricoles de la fin du XIX siècle, la crise boursière des années trente. La maison, celle du médecin, reste le dernier refuge, à préserver à tout prix.

La première des filles du médecin, née en 1899, sera la mère de Michel Rochefort.

En 1902 s'installe à Joux le premier instituteur de l'école laïque. Auparavant, des religieuses éduquaient les enfants, du moins ceux dont les parents pouvaient se l'offrir. L'instituteur fit sa visite de courtoisie au médecin qui le reçut et le raccompagna à la petite porte. Les filles du médecin étaient pensionnaires à Sainte Jeanne d'Arc à Avallon, et il n'était guère question de frayer avec le diable en ces temps troublés de séparation de l'Église et de l'État. Médecin et instituteur se retrouveront cependant plus tard dans un commun amour de la chasse, du bridge et du tarot. Une vie sociale ne pouvait que se nouer au sein de la si petite élite non-paysanne du village. Et c'est ainsi que le fils de l'instituteur épousa la petite-fille du notaire pour devenir le père de Michel Rochefort.

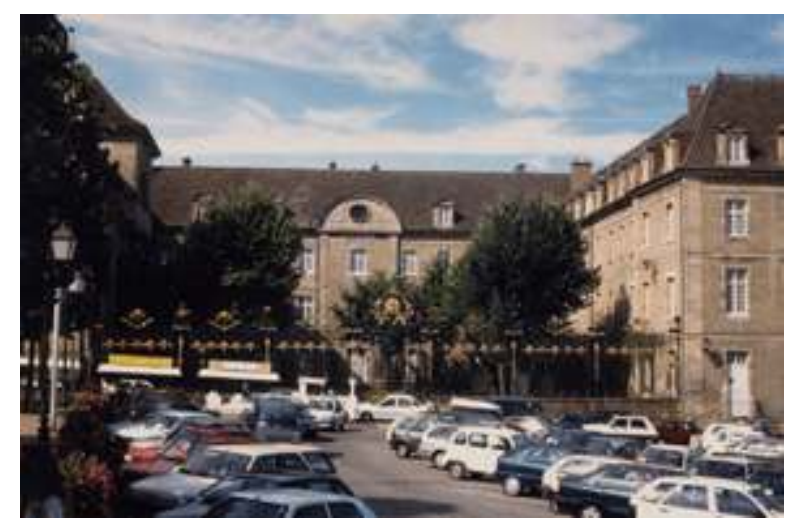

Le lycée Bonaparte d'Autun. Photo Françoise Plet, 1998.

Après la guerre qu'il fit en 1918, le jeune Henri Rochefort, poursuivant une ascension classique des familles enseignantes devint professeur de philosophie au lycée Bonaparte d'Autun où se déroula toute sa carrière, avant de prendre sa retraite à Joux, dans la maison natale de sa femme, qui fut aussi la maison natale de chacun de ses trois fils. La vie de travail et Autun étaient pour lui une sorte de parenthèse. Il était, selon d'anciens élèves que j'ai pu rencontrer, un professeur très apprécié qui savait d'instinct faire comprendre et aimer la philosophie, et assurer à sa classe parmi les meilleures 
notes au baccalauréat. Mais, hors les grilles du lycée, la vie sociale était limitée. Jamais il ne s'est adapté à la vie de la petite ville. Autun et sa région étaient visités comme une terre étrangère. Les samedi et dimanche, c'était la pêche au lac des Settons, ou bien, l'hiver, des journées en forêt de Planoise ${ }^{8}$ où les particuliers pouvaient acheter sur pied et couper eux-mêmes leur bois de chauffage. Les vacances se déroulaient à Joux. Le professeur avait assez mal vécu sa situation de pensionnaire au lycée d'Auxerre, et sans doute même sa vie d'étudiant à Paris. L'homme était un rural profond, solitaire, aimant la forêt. Michel Rochefort évoque un bout de film familial où on le voit s'éloignant seul en direction d'un bois.

18 C'est dans ce cadre que se déroulent l'enfance et l'adolescence de Michel, jusqu'à ses 17 ans. Une adolescence marquée par les difficultés, les privations et les drames personnels de la guerre. Autun était en zone occupée. Des copains résistants sont morts. Rude baptême de la vie qui se poursuit à Paris à partir d'octobre 1944 car les conditions de vie y sont dures, favorisant sans doute la tuberculose découverte en 1947.

Géographe étudiant des terroirs qui lui sont proches, Michel Rochefort l'est devenu au hasard de la maladie. Jeune homme, il est en classe préparatoire à Paris avec l'objectif d'entrer à l'École Normale Supérieure, en philosophie, sur les traces du père, poursuivant la voie de la progression de la branche paternelle dans le cursus établi de l'excellence professorale. Mais au lieu du concours, ce sera le sanatorium. Michel Rochefort qui avait fait beaucoup d'histoire en hypokhâgne et khâgne, notamment en rapport à la philosophie de Hegel, termine donc une licence d'histoire. Jean Tricart, alors assistant d'André Cholley à l'Institut de géographie de Paris, l'aide dans l'apprentissage de l'explication des cartes géologiques nécessaire à l'obtention du certificat de géographie, et finalement à se convertir à la géographie.

Michel Rochefort n'est plus retourné à Autun après son Diplôme d'Études Supérieures (DES) ${ }^{9}$, réalisé sous la direction de Jean Tricart et soutenu à Strasbourg en 1949, même si l'année suivante, grâce à une bourse du CNRS, il a étudié «le bocage récent d'Allerey » dans le pays d'Arnay-le-Duc, en Auxois, toujours à proximité de l'une des routes qui relient Autun à Joux ${ }^{10}$ (1950). C'était juste avant l'agrégation obtenue en 1951, avant son mariage et le début de sa thèse sur L'organisation urbaine de l'Alsace. Ce sera sa dernière publication relative à un territoire rural, même s'il mène une observation continue de son terroir de Joux.

21 Car Joux est resté son havre, le lieu de mémoire et la maison, l'unique bien qu'il s'est ingénié à conserver. "Toutes les vacances se passaient ici. Mon père adorait les bois, et s'en occupait, voyait ce qu'il fallait couper, coupait lui-même. Donc on a beaucoup vécu dans les bois, on allait chercher des champignons. Et quand il a pris sa retraite, il s'est installé ici ». Michel Rochefort y revient régulièrement, discute avec le maire des affaires du pays, observe et se promet, lorsqu'il aura un peu plus de temps, de fouiller les archives, de reprendre et d'approfondir l'enquête sur le passé de cette société locale inscrite à Joux.

Joux d'un siècle à l'autre sous le regard de Michel Rochefort

Dans Un exemple de l'influence des nouvelles techniques agricoles sur un budget paysan ${ }^{11}$, il compare des budgets d'exploitation à Joux vers 1890 "grâce aux souvenirs de deux paysannes de 74 et 75 ans, un paysan de 75 ", et en 1949, sur une exploitation de taille équivalente à la première étudiée. Il y constate que "la transformation des techniques (engrais, machines) [...] qui augmentent les rendements, permettent la culture des plantes sarclées, [et celle] des fourrages artificiels qui entraîne un élevage bovin important [qui ont produit] une augmentation considérable de la rentabilité du travail, [de celle] de la terre pour 
l'exploitant [...] une diminution de la densité rurale [ce qui se] traduit à Joux-la-ville par le départ de tous les journaliers qui vivaient jadis en travaillant trois mois pour un cultivateur, trois mois pour un vigneron ... et six mois comme bûcherons ». Mais, ajoute-t-il quant à la rentabilité de la terre, «les chiffres pour la grande propriété domaniale paraissent devoir être différents ».

Sont ainsi campés ses informateurs familiers, son sens aigu des disparités sociales et de leurs évolutions relatives, des effets sur les collectivités de changements individuels liés à l'environnement économique global. La grande propriété domaniale, c'est l'exemple de sa famille à laquelle la terre n'assure plus aucune ressource. Les paysans d'autrefois, il s'en fait une connaissance intime grâce à la bonne et au jardinier du grand-père médecin, qu'il interroge sans relâche afin de savoir ce qui existait "avant ". Ils s'y prêtent avec plaisir et empressement : "Attends, je vais demander » et s'en vont discuter au village afin de préciser les réponses à ses questionnements. C'est sa ressource depuis toujours pour pénétrer une intimité populaire dans une société villageoise dont il est membre et où la coupure est étanche entre la bourgeoisie des professions libérales et intellectuelles, et le monde des commerçants et paysans, la distance étant soigneusement maintenue, indépendamment de la fortune de chacun. Jeanne, la bonne était née en 1873. Seconde fille d'un petit vigneron agriculteur, elle avait sept ans lorsqu'est née la fille du notaire que sa mère, bourgeoise de Vitteaux, voulait élever avec une aide. C'est ainsi que la petite Jeanne est devenue la servante de la future épouse du médecin et n'a plus quitté la famille durant le reste de ses 92 années de vie. Le jardinier, lui, était fils de manouvrier.

$\mathrm{Au} \mathrm{XIX}^{\mathrm{e}}$ siècle et jusqu'à la mécanisation, la vie était très dure sur ces plateaux rocailleux et froids qui usaient serpes et bras pour de tout petits rendements (dix quintaux de blé à l'hectare) ${ }^{12}$. Sur les pentes internes de la cuvette était la petite propriété paysanne, avec des champs étroits et allongés, de deux mètres de large près $\mathrm{du}$ village, lanières qui se perçoivent encore à la forme des quelques boisements dispersés qui subsistent çà et là. Dans les vallées sèches qui descendent vers la vallée de la Cure étaient les vignobles. La moitié de la population du village était vigneronne, dans les hameaux et au bourg. Michel Rochefort montre au sein du bourg la place importante dans le bâti des anciennes maisons de manouvriers ou de vignerons paysans qui avaient des terres pour se nourrir. Rétif parle des vins de Sacy et de Val-deMalon, qui est un hameau de Joux. Tout autour du finage villageois, sur le plateau, des hameaux de grosses fermes avec leurs ouvriers. À l'Est, la grande forêt d'Hervaux ( 3000 ha) forme la frontière du finage, avec le Val-de-la-Nef, ferme clairière de 300 ha et hameau de bûcherons. Vignes et agriculture manuelles étaient peuplantes. Joux comptait encore 1400 habitants vers 1890 et 1200 en 1900, tandis que le phylloxéra exerçait ses ravages.

Nicolas Restif de la Bretonne montre l'achèvement de ces finages bourguignons dans la première moitié du XVIII siècle en insistant sur l'importance de l'initiative de paysans aisés arrondissant leur bien et diversifiant les productions ${ }^{13}$. "Arrivé sur le terrain âpre et stérile de Sacy, la vue de ces champs blanchis de pierres et brûlés par le soleil, les cris sourds et inarticulés des pesans laboureurs qui luttaient contre la nature et la voulaient forcer de les nourrir, jetèrent [la tristesse] dans son âme ». Sur la ferme de son beau-père, il épierre : "Edmond sacrifia le haut du champ, presque absolument non labourable, pour y amonceler les pierres... Il eut soin de massoner lui-même, avec les pierres les plus larges le bas du merger (c'est le nom que l'on donne à ces tas de pierres) et de mêler dans les entre-deux un peu de terre avec 
des touffes de laume et d'autres herbes du genre des graminées, jusqu'à hauteur d'un homme, tant pour consolider par là le bas du merger, que pour fournir une pâture aux bestiaux presque égale en étendue, au terrein qu'il était forcé de couvrir. Il avait aussi eu soin de pratiquer un chemin en limaçon pour monter jusqu'au sommet et chaque année, avant le labourage, on y portait les pierres que les pluies avaient découvertes ». Le beau-père s'oppose à la poursuite de l'expérience, mais les autres paysans, eux, l'imitent et «le sommet aride des collines fut couronné de mergers immenses et les champs voisins commencèrent à produire ».

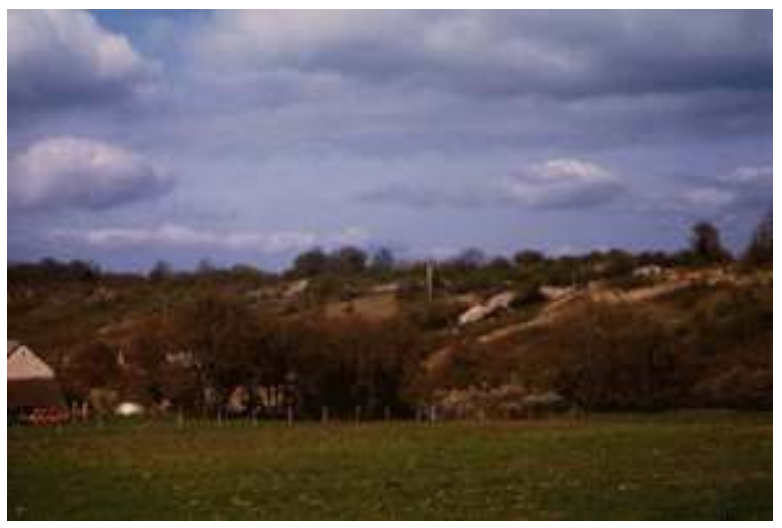

Murgers à Joux. Photo Françoise Plet, 1999.

Puis il s'attaqua au défrichement des incultes «qui formaient bien le tiers du finage», les laissant en fourrage la première année, puis leur appliquant trois labours avant de produire un blé. "Épines et genévriers [fournissent un] ... excellent chauffage pour le four à cuire le pain ». Mais il est des pentes indéfrichables car elles sont trop raides. Edme Rétif y plante de la vigne qui met sept ans avant de produire. "Mais enfin elle produit un vin excellent, qui n'avait d'autre défaut que d'être trop tendre, c'est-à-dire potable au bout de six mois, et ne pouvant se garder au delà de trois ans dans toute sa bonté. À son imitation le laborieux saxiate $e^{14}$ planta des coteaux incultes, et bientôt le produit des vignes, absolument créé puisqu'il n'existait pas auparavant, surpassa celui des terres... Il fallut environ trente ans pour donner à cette culture le degré de perfection et de rapport qu'elle a aujourd'hui» (1779). «En diminuant les friches, on avait augmenté les bestiaux nécessaires à la culture; devenus plus aisés, les habitans s'étaient donné les utiles animaux qui adoucissent la vie, la vache, la brebis, la chèvre: on avait besoin d'une plus grande quantité de fourrage, et par un retour profitable à l'agriculture, cette consommation produisait une plus grande quantité d'engrais". Cette révolution agricole, si l'en on croit Restif, se situe entre 1720 et 1750. Et Edme s'est créé son propre domaine, le "domaine de la Bretonne, qui est absolument isolé, et ne touche au village que par les murs d'un enclos assez étendu ». Tout est ici campé pour la fin du siècle suivant, le village, le grand domaine extérieur, le vignoble. Et en filigrane la place essentielle des journaliers toutes mains dont l'année de travail est si précisément retracée par Michel Rochefort.

Dans cette société de petits paysans et d'ouvriers agricoles vivant des céréales, de la vente du vin et de quelques moutons, les vignerons agriculteurs sont ruinés par le phylloxéra et achevés par la crise de mévente des céréales de la fin du XIX ${ }^{\mathrm{e}}$ siècle. Ils émigrent, à Paris surtout. La friche regagne les vallons dont les hameaux sont dépeuplés ou abandonnés. De mauvais bois ou des plantations de pin noir pour la chasse remplacent le blé sur les lanières abandonnées du terroir villageois. La Grande guerre parachève le déclin. Les noyers qui parsemaient l'openfield et procuraient 
l'huile n'ont pas attendu la grande mécanisation pour presque totalement disparaître : ils ont servi à fabriquer des crosses de fusils.

Les habitants qui restent élèvent des chèvres et rachètent des friches. Les propriétaires des grandes fermes les vendent, ainsi la ferme d'Oudun, vendue en 1928 à un vétérinaire, qui a conservé les mêmes fermiers jusqu'en 1947, date à laquelle ceux-ci l'ont achetée, puis agrandie : sa surface est passée de 150 à 200 ha. Mais si l'on en juge aux exemples donnés par Michel Rochefort, ni la part respective du faire-valoir direct et indirect, ni la surface de l'exploitation moyenne n'avaient beaucoup varié de 1890 à la fin des années quarante ${ }^{15}$. C'est la productivité du travail qui s'était améliorée, et le produit à l'hectare. Plus aucun journalier, et pourtant au travail de la terre s'ajoutait un troupeau de laitières, tandis que le mouton avait disparu.

Ce sont ces plateaux bourguignons qui furent à la fin des années 1950 l'objet des actions de la Société des "Friches et taillis de l'Est ", avec un premier remembrement d'autant plus réussi que la conjoncture agricole était favorable, et les techniques de mieux en mieux adaptées à la production sur terres difficiles. À partir des années 70 surtout se multiplient les défrichements de bosquets qui accompagnent l'expansion de l'espace cultivé et la concentration des exploitations agricoles. Un bois de 50 hectares, dernier vestige des biens fonciers de la famille Lecourt-Rochefort, une fois vendu, a été défriché par le nouveau propriétaire. Un récent remembrement (1995) entérine la concentration spontanée et accélère la disparition des petits bois. Plusieurs chantiers de défrichement étaient en cours dans le finage à l'été 1998. Il reste au bourg cinq gros propriétaires, exploitant de 200 à 300 hectares, tous issus de vieilles familles agricoles locales. Propriété et exploitation sont aujourd'hui en grande partie conjointes. À côté de la grande culture stricte cependant s'observent des diversifications telles un élevage laitier, une production de porcs de plein air, ou la vente de produits fermiers. Des bûcherons de la forêt d'Hervaux, il en reste deux qui veillent jalousement à leur quasimonopole de la cueillette des champignons.

Le village à première vue n'a guère connu de construction récente. Point de lotissement massif en bord de route, ni de ces maisons cubiques caractéristiques des années 50 . Mais si l'on s'enfonce dans les ruelles, on observe partout reprises et rénovations pour l'habitat, près de la mairie, près de l'église, à l'est de la grande rue tandis qu'une dizaine de maisons neuves s'alignent sur la route de la forêt, petit lotissement qui accueille surtout des retraités de l'agriculture après la cession de leur exploitation. Retraités et vacanciers ont repris des maisons vacantes, au bourg et dans les hameaux : ouvriers émigrés à Paris et enfants d'émigrés, étrangers, Néerlandais notamment. Val-de-Malon par exemple, un ancien hameau viticole, a été entièrement restauré.

$\mathrm{Au}$ milieu des années 80 , le centre de détention nouvellement construit, centre de fin de peine, devient un élément supplémentaire du repeuplement du village, passé de 450 habitants en 1950 à 530 en 1998. Gardiens et personnels de service viennent résider à Joux où la mairie leur propose des logements rénovés par ses soins. Un ensemble de services à la population s'est implanté ou réimplanté au village après une période de déclin. Cinquante ans après la mort du grand-père médecin, en 1988, à nouveau un médecin exerce à Joux, lequel a curieusement retrouvé l'aire de clientèle de six villages qui fut celle du grand-père, effet sans doute de centralité structurelle ancienne qui minimise les distances. Car Joux est au centre d'une étoile de routes, tandis que les structures de la circulation sont plus linéaires au long de la vallée de la Cure. Des kinésithérapeutes se sont également installés. Un garage se maintient. Une boulangerie 
remplace le dépôt de pain à l'été 1998. Un processus qui autorise Michel Rochefort à s'élever vigoureusement contre l'idée de "désertification rurale» si complaisamment véhiculée dans certains milieux, y compris parmi les géographes.

L'évolution des détenteurs de la notabilité locale a accompagné l'évolution de la société et des activités du village. Au cours du siècle, la mairie a été successivement tenue par un notable, l'arrière-grand-père notaire, puis par un instituteur retraité de 1935 à 1945. À cette date, c'est un gros propriétaire de la mouvance des Indépendants16, qui prend la mairie. En 1982 c'est un maire de gauche qui est élu, témoin à la fois du changement politique global, et du changement local dans la population, dont une partie est salariée et travaille à Avallon, à ${ }^{16} \mathrm{~km}$ de là. Le nouveau maire est issu de l'assistance publique, a été élevé par une famille du village, et conduit une petite entreprise du bâtiment. C'est lui qui a accueilli sur sa commune la prison, planifiée par le gouvernement Chirac, et refusée par les habitants de la commune voisine dont le maire et conseiller général appartenait pourtant à la majorité d'alors. La peur des nuisances chez lui l'a emporté tandis que le maire de Joux a choisi d'accepter l'implantation, et d'en gérer la localisation et les impacts. Nichée aux limites de la commune, invisible du village, elle apporte des revenus fiscaux et une population pour laquelle la municipalité a aménagé des logements au centre du village, sur une partie des terrains du parc de la mairie.

Le ruraliste des premiers travaux : une approche historienne des structures sociospatiales

La "méthode Rochefort » des années 50 est marquée par le recours au passé, à une histoire longue dans une recherche de l'intelligence de l'organisation des finages et de leurs paysages, à travers l'étude de l'évolution des structures sociales et des rapports sociaux et économiques, bref de la société avec son épaisseur historique. Les problématiques sont toujours fondamentalement celles de l'origine des structures agraires observables, qu'il s'agisse du bocage d'Allerey ${ }^{17}$, ou de la comparaison entre Autunois, Morvan et haut plateau gréseux de l'est d'Autun qui fait l'objet du DES et des publications qu'il en a tirées ${ }^{18}$. Il est en cela proche des travaux de l'époque qui mobilisaient un important appareil d'archives pour une analyse rétrospective, et en accord avec son inclination pour l'histoire.

S'y ajoute, pour ce qui concerne l'Autunois surtout, la thèse des effets différenciés de l'inégale pénétration des capitaux de la bourgeoisie urbaine dans les campagnes, qui commençait alors à être développée autour de Pierre George ${ }^{19}$, et de manière plus générale, celle de l'influence de la propriété foncière sur l'exploitation agricole, avec l'idée sous-jacente, que le faire-valoir direct favorisait l'initiative agricole et le «progrès »20. Enfin il y joint l'attention aux effets massifs des conjonctures économiques que commençaient à développer les historiens autour de Labrousse.

Le bocage d'Allerey (Auxois) - marché et capitalisme agricole

Par exemple l'étude sur le bocage d'Allerey, souvent citée, décrit en 1950 en Auxois une structure de grandes exploitations isolées à niveau de vie très élevé, avec des prés massifs entourés de haies et de grands champs labourés. Partant du constat qu'il s'agissait au XIXe siècle d'un openfield labouré avec pacage collectif de moutons comme dans son pays de Joux, il explique l'évolution par l'avantage économique procuré par l'élevage bovin à partir de 1860, qui s'est accompagné d'un grand mouvement d'appropriation foncière par les plus aisés des paysans, de l'agrandissement des exploitations agricoles, et du développement de la mise en herbe et des clôtures encore 
actif au moment où il écrit. En bref un effet du marché qui favorise le développement d'un capitalisme agricole.

Plaine d'Autun et bordures morvandelles : l'inégale pénétration des capitaux bourgeois

Michel Rochefort part du contraste observé entre «la très grande prédominance du fairevaloir indirect et des fermes isolées au milieu de vastes parcelles irrégulières et entourées de haies [de l'Autunois] qui s'opposent aux hameaux de propriétaires exploitants du Morvan et du plateau, aussi bien qu'à leurs champs étroits et allongés ».

Il mène ensuite une longue enquête dans les archives et les ouvrages pour constater que "partout la société féodale avait projeté sur la terre l'unité de sa structure sociale ». C'est aux XVII ${ }^{e}$ et XVIII ${ }^{e}$ siècles que les capitaux autunois, mettant à profit la misère rurale et les embarras financiers des seigneurs, se taillent des domaines dans la plaine. Au premier cadastre (1825-1829), "90\% de la surface cultivée [...] est occupé presque exclusivement par des domaines" tandis qu'en Morvan, "les domaines voisinent avec de nombreuses agglomérations paysannes». Cette structure domaniale s'indure ensuite en Autunois à la faveur de l'amendement des sols par chaulage dans la seconde moitié du $\mathrm{XIX}^{\mathrm{e}}$ siècle qui améliore les revenus des partenaires, fermiers et propriétaires, en dépit de rendements très faibles (six quintaux de blé à l'hectare tandis qu'on en produisait dix à Joux à la même époque). La crise céréalière, ici apparemment en 1905, et l'émigration de la main-d'œuvre agricole, orientent les propriétaires vers la mise en prairies. La population agricole passe de ${ }^{20}$ à 9 habitants par $\mathrm{km}^{2}$. Quelques domaines sont démembrés, mais en 1948 «les propriétaires bourgeois et nobles occupent encore $81 \%$ des terres ». Des propriétaires exploitants sont apparus, tandis que " la reprise du domaine par le propriétaire en faire-valoir direct s'accentue ", ce qui s'expliquerait par "le manque de bras», les hommes préférant "au prolétariat rural [...] le prolétariat urbain». À cette fragilisation somme toute relative du domaine, Michel Rochefort oppose une situation dramatique du Morvan, tandis que «le haut plateau triasique $e^{21}$ a ... retrouvé un équilibre, car la proximité de la grande usine du Creusot permet au paysan d'aller comme ouvrier gagner l'argent qu'il faut ajouter pour vivre au produit de la petite propriété cultivée par la femme, [...] le Morvan se meurt et n'évitera sans doute la dépopulation presque totale que par une organisation des exploitations indépendante des structures de la propriété».

Un demi-siècle plus tard, à travers d'autres conjonctures... ${ }^{22}$ L'effacement de l'influence de la nature et de la structure de la propriété foncière sur les exploitations agricoles

Si j'interroge aujourd'hui Autunois, Morvan et plateau, ce sont les ressemblances agricoles qui me frappent, quelles que soient les structures foncières sous-jacentes. Partout des exploitations plutôt grandes, individuelles ou en $\mathrm{GAEC}^{23}$, qui produisent des bovins de race charolaise pour la viande, avec un système de culture fondé sur l'herbe, des prairies régulièrement resemées, et quelques cultures énergétiques pour l'alimentation d'hiver, maïs et triticale, qui représentent à peu près $20 \%$ des surfaces utilisées. Une sélection s'est opérée en faveur des plus gros des éleveurs de 1948 qui ont concentré l'utilisation des terres à leur profit. Cette concentration agricole a été en cinquante ans nécessairement beaucoup plus forte en Morvan et sur le plateau gréseux. Mais dans tous les cas, elle s'est réalisée, comme Michel Rochefort le jugeait nécessaire en Morvan, par un affranchissement des exploitations agricoles vis-à-vis des structures de la propriété.

Ces structures foncières restent différentes. À Roussillon-en-Morvan, le parcellaire cadastral reste émietté. La mentalité «bien de famille » perdure en dépit du fait que beaucoup de propriétaires ne sachent plus où se trouvent leurs parcelles, qui sont 
effectivement utilisées par d'autres. On a proposé des échanges gratuits de terre pour remembrer, mais cela n'a pas marché. Ce sont les agriculteurs qui ont opéré entre eux leurs propres regroupements, indépendants de la propriété. Parallèlement, les mailles du bocage ont été considérablement élargies car les propriétaires ne peuvent obliger quelqu'un à cultiver de petites parcelles. La municipalité y a aidé. Les années 50 avaient été celles de l'augmentation de la proportion de forêt. Le développement anarchique des plantations de sapins sur de petites parcelles enclavées ont conduit la commune, dans les années 70, à interdire tout boisement à moins de cinq mètres des limites des parcelles agricoles, ce qui a limité la fermeture et le mitage des paysages. En dépit du développement de la location, la propriété reste dominante parmi les terres des exploitations agricoles en activité.

41 À Monthelon au contraire, les propriétaires ont souvent vendu leur ferme à leur fermier. La pratique s'est développée à partir de 1975-80, notamment en fin de bail, ou lorsque le fermier partait à la retraite. Le prix des terres agricoles atteignait alors les plus hauts niveaux jamais connus avant d'amorcer une chute brutale et profonde à partir de 1980. Les lois de 1945 et 1946 portant statut du fermage et du métayage, lesquelles donnent droit de préemption au fermier en cas de vente des terres restaient difficiles à appliquer en 1948 par crainte des propriétaires et par manque de liquidités financières. La création des Sociétés d'Aménagement Foncier et d'Établissement Rural (SAFER) et la réglementation des cumuls d'exploitations mises en place par la loi de 1960, qui limitent le libre jeu de l'offre et de la demande, le développement des prêts à faible taux d'intérêt ${ }^{24}$, ont rendu plus faciles les achats des fermiers. En 1998 encore, une propriété de 300 ha a été vendue. De sorte qu'à Monthelon aussi, presque tous les exploitants sont à la fois propriétaires et fermiers, mais avec un taux de propriété de $30 \%$ en moyenne, ce qui reste très inférieur à ce qui existe à Roussillon-en-Morvan où la part en faire-valoir direct dépasse largement $50 \%$ des surfaces exploitées. Et si les bailleurs sont tous en Morvan de famille paysanne et issus du village, ceux de Monthelon demeurent extérieurs à la commune. Ce sont des ingénieurs, des retraités, un pharmacien de Dijon, un boucher d'Étang-sur-Arroux, un entrepreneur, des exploitants agricoles, un propriétaire d'immeubles à Beaune, un propriétaire de vignobles à Auxey-Duresses... Ils ont une «mentalité foncière» selon le maire de Monthelon, mais ils ne sont plus du tout Autunois.

Les petites et moyennes exploitations agricoles ont quasi disparu, même s'il en existe encore quelques-unes à Roussillon. Les plus grandes ont évolué de manière homothétique à leur taille d'origine, de sorte que leurs surfaces restent sensiblement plus petites en Morvan. Cependant, les systèmes de production, et la taille des cheptels sont devenus les mêmes. Cette plus grande intensivité à l'Ouest est en grande partie liée aux avantages pédo-climatiques du Morvan par rapport aux collines du bassin d'Autun où le sol est très boueux lorsqu'il pleut, où la sécheresse d'été ne favorise pas l'herbe sur les limons siliceux, et où l'eau manque sur les pâtures des versants de sorte que des compteurs de prés y ont été installés à partir de l'adduction communale afin d'abreuver les animaux. Et l'on approvisionne en nourriture quand il fait sec. Le Morvan est moins sensible à ces aléas.

À Roussillon, jusqu'aux années 50, les exploitations étaient petites, de trois à quatre vaches, quelques-unes seulement en ayant de dix à quinze. La double activité bûcheron et agriculteur n'était pas rare. À partir de 1953, tout change très vite avec les tracteurs. Si l'on cultive encore blé, seigle et pomme de terre, une variété demi-sauvage ${ }^{25}$ qui 
résiste ici plus longtemps que dans la plaine, le tracteur permet le semis de prairies. Les grosses exploitations avaient jusqu'en 1968 autour de 30 ha, 15 vaches, et produisaient encore du lait pour la moitié du cheptel, des veaux de boucherie et des animaux gras. Depuis, l'augmentation de la charge bovine a été très forte. Il reste en 1998 à Roussillon 14 exploitations agricoles dont 9 ont un nombre économiquement viable de bêtes. Celles qui comptent sont des GAEC de 50 à 60 ha, élevant de 170 à 243 bêtes.

De la même manière en Autunois, les micro-domaines complémentaires des artisans ont disparu les premiers, puis dans les années 70, les petites exploitations de 6 à 16 ha. L'évolution s'est également faite sans remembrement. Il y a en 1998 à Monthelon 20 grandes exploitations. Elles étaient 36 en 1950. Les fermes regroupées ont repris les autres par achats ou locations, ce qui a fait passer récemment ${ }^{26}$ les surfaces moyennes de 55-60 ha à 150-160 ha. Les regroupements ont commencé vers 1975, initiés par les jeunes agriculteurs d'alors. La densité bovine approche de deux bovins/ha. Elle est donc beaucoup plus faible qu'à Roussillon où le cheptel gros bovins total ${ }^{27}$ a plus que doublé entre 1968 et 1991.

Le système de production est partout le même. Les pâtures épuisées sont fauchées, puis labourées pour produire du maïs trois ou quatre ans avant le resemis de prairies. Le mouton, un temps important, est encore élevé dans cinq des exploitations de Roussillon, une seule à Monthelon. Les laitières ont disparu dans les années 60 , après la suppression de la collecte du lait. La spécialisation viande bovine l'a emporté, excluant pour un temps tout autre débouché marchand.

L'élevage est naturel, lié aux saisons d'herbe et aux exigences de la Politique agricole commune (PAC), parfois en insémination artificielle grâce à laquelle la qualité cheptel a été améliorée. Mais l'on n'est pas ici dans une zone de sélection de bêtes à concours, ou de génétique sophistiquée avec transferts d'embryons et vaches porteuses. Le changement du début des années quatre-vingt a été le passage à la vente en maigre. Plus personne n'engraisse à Roussillon, et trois exploitations seulement engraissent une partie de leur cheptel à Monthelon. Le naissage continue d'être groupé en décembre. Il y a eu des tentatives de changement de rythme, avec des inséminations précoces pour avoir des veaux à partir de novembre. Mais les primes européennes ${ }^{28}$, qui exigent des carcasses légères, ont fait reporter les naissances pour mettre les veaux au pré plus jeunes, annulant les velléités de désaisonnement engagées dans le but de profiter des périodes de cours élevés. Personne ici n'est intéressé par les primes régionales créées pour favoriser l'engraissement sur place.

Dans les deux cas, la coopération et l'entraide entre exploitations jouent un rôle important, quoique très récent. À Roussillon, deux CUMA ${ }^{29}$ sur plusieurs communes ont été créées au milieu des années quatre-vingt, "plus tard qu'à La-Celle-en-Morvan ou Monthelon, car en bas il y a beaucoup plus de fermiers de gros propriétaires, et plus de besoins en matériel ». Deux CUMA aussi fonctionnent à Monthelon, qui sont moins précoces que celles de Bresse et du Charollais ${ }^{30}$. Elles servent pour le gros matériel de semis, de récolte et d'ensilage, réduisent les investissements de chaque exploitation et pallient en partie une main-d'œuvre réduite aux seuls chefs d'exploitation ou associés des GAEC. Il n'est pas rare chez ces éleveurs en moyenne jeunes qu'une seule personne s'occupe de 100 ha et 150 animaux avec la seule aide occasionnelle du père retraité.

Les réseaux de commercialisation sont différents dans chacune des zones, ce qui traduit surtout des différences en termes d'aires de relations. En Morvan, les bêtes sont vendues au marché au cadran de Moulins-Engilbert dans la Nièvre, ou bien à des 
marchands belges ou hollandais qui passent dans les fermes, tandis qu'à Monthelon, les acheteurs sont des rabatteurs locaux pour l'Italie ${ }^{31}$ et le Groupement de producteurs de Charolles en Saône-et-Loire, avec une progression récente de la SICA 21 de Côte-d'Or, car chaque structure de commercialisation cherche à augmenter ses volumes afin de pallier la faiblesse des cours ${ }^{32}$ qui met en difficulté les finances des groupements.

L'influence des structures foncières héritées dans les différenciations rurales récentes La société rurale, elle, reste différente selon les zones. Elle a changé sous d'autres contraintes que la contrainte foncière. Le Creusot n'est plus pourvoyeur d'emplois pour ouvriers-paysans. Mais la proximité de la ville d'Autun, les structures foncières héritées et l'organisation ancienne des finages décrite par Michel Rochefort jouent encore. Roussillon est devenu plus nettement un Morvan autunois par la structure de la population et celle des emplois qu'elle exerce. Monthelon, la plus domaniale des communes de la plaine étudiées par Michel Rochefort, change à peine, tandis que les hameaux de Tavernay - ceux des anciens petits domaines paysans - sont occupés par des employés et ouvriers d'Autun, que Saint-Forgeot est pour partie, avec ses grands ensembles, ses surfaces commerciales et sa zone d'activités, agrégé à la ville d'Autun.

À Roussillon-en-Morvan, la population permanente est stabilisée à 339 habitants, contre 500 dans les années 50. L'émigration y a été importante dans les années 60/70, celles de la concentration des exploitations agricoles, lorsque les jeunes nés dans l'après-guerre ont été en âge de partir. Elle s'est faite surtout vers Paris. Parallèlement s'est développée la résidence secondaire: le tiers des maisons sont des résidences secondaires ce qui augmente considérablement la population en été. Une part de ces maisons rénovées des hameaux appartiennent à des Hollandais ( 25 familles), des Belges, des Anglais, dont certains sont des résidents permanents. La situation au sein du Parc naturel régional du Morvan, comme les paysages, ont ici un effet certain. Plus traditionnelles, deux colonies de vacances fonctionnent dans la commune. Entre agriculteurs, employés migrants pendulaires vers Autun, retraités, et fréquentation touristique, le bourg comme les hameaux de Roussillon n'ont plus rien de ce paysage d'extrême pauvreté qu'ils offraient à la fin des années quarante.

51 Monthelon au contraire, pourtant plus proche d'Autun, conserve une tonalité profondément agricole. La population y avait encore diminué au recensement de 1990. Les 250 habitants sont à $40 \%$ agriculteurs, à $30 \%$ retraités de l'agriculture. C'est en 1997 seulement qu'un changement s'amorce avec quatre maisons neuves dont trois abritent des ménages venant des HLM d'Autun, et la création d'un lotissement communal de huit logements. Les agriculteurs étant opposés à la vente de parcelles pour la construction, ce qui indique leur force, le maire a réalisé son lotissement sur une partie des communaux, le reste en étant attribué à un éleveur. Les ressources fiscales de la commune sont exclusivement les taxes foncières et les taxes d'habitation, à l'exception de la contribution d'une petite installation d'EDF. Seuls, un gîte rural, trois chambres d'hôte et une auberge à la ferme qui ne fonctionne que les dimanches de mai à octobre, représentent une très modeste diversification agro-touristique.

52 C'est donc aujourd'hui par la place et les poids respectifs de l'agriculture, des autres activités des résidents permanents, et le rôle des résidents temporaires dans la vie des communes plus que par les différences de structure des exploitations agricoles que se manifeste encore l'effet des caractéristiques foncières héritées de la période de "pénétration des capitaux bourgeois dans la campagne autunoise». Et comme à Joux, les 
maires représentent bien cet état différent des lieux : à Roussillon, il s'agit d'un cadre commercial, à Monthelon d'un retraité, ancien éleveur et propriétaire forestier.

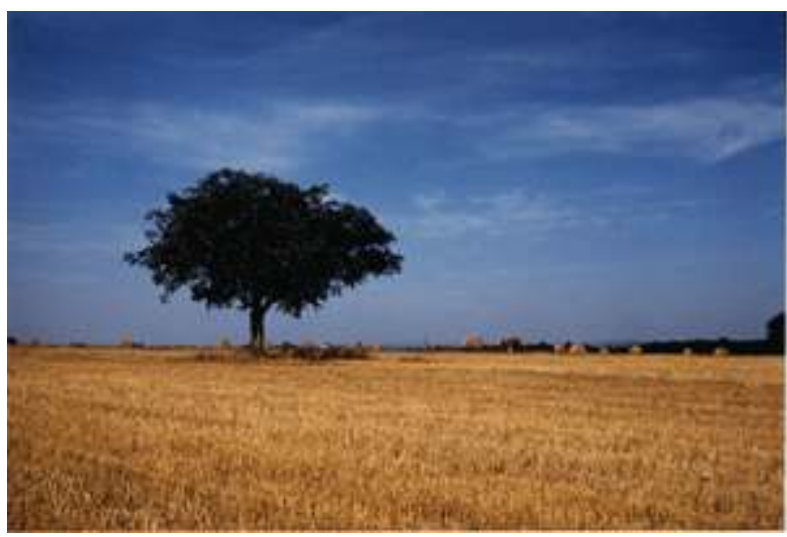

Paysage à Joux-la-ville. Vestige de l'ancien openfield à noyers. Photo Françoise Plet, 1998.

\section{ANNEXES}

La maison de Joux

À la mort de sa mère en 1982, aucun des deux frères de Michel Rochefort ne souhaite conserver une part de la maison de Joux reçue en héritage. Il ne peut matériellement la reprendre seul en leur versant les soultes indispensables. Mais, comme ses parents, comme sa grand-mère, il veut de toutes ses forces la conserver. Il imagine alors la création et la gestion d'une co-propriété complexe en trois lots mais une trentaine de parcelles de manière à garantir chacun en cas de rupture d'entente, avec espaces communs et espaces privés, dépenses partagées et dépenses individuelles. Deux amis sûrs sont devenus ses co-propriétaires. Ainsi se perpétue une présence, avec des réunions familiales où les trois frères aiment à se retrouver, ses filles et leurs amis qui y prennent leurs habitudes, ses petits-enfants. C'est le lieu des proches, les vivants, et tous les autres. L'acte signe aussi une personnalité d'organisateur et de gestionnaire passée un peu inaperçue tant elle s'exerce avec une efficacité discrète, dans des interstices, en marge des premiers rôles universitaires et des effets d'affiche.

La Bourgogne à Paris : la confrérie des Bourguignons salés

Au-delà du village, la Bourgogne de Michel Rochefort aujourd'hui, c'est le rayon des promenades à vélo, "ces chemins dont il connait chaque caillou ", les vins et la gastronomie - la belle cave de la maison est soigneusement tenue à l'abri de la lumière et alimentée avec goût - et les « Bourguignons salés ».

Exclusivement masculine, cette « Académie culturelle » réunit des Bourguignons habitant Paris et bénéficiant de suffisamment de quartiers de burgondité, soit au moins des parents et grands-parents bourguignons. Elle est limitée à 50 membres renouvelés par cooptation de deux parrains membres actifs lorsque des titulaires font défaut, soit 
par décès, soit par perte de leur qualité de membre s'ils ne participent pas à au moins deux des dîners mensuels. Ces dîners se déroulent d'octobre à juin et l'on y déguste un vin blanc et un vin rouge de Bourgogne, tirés de la cave collective de la confrérie. Des femmes n'y sont admises qu'une fois par an à un « dîner des dames » auquel s'ajoute un déjeuner d'été en terre bourguignonne.

Fondée il y a un siècle, la confrérie comprenait alors beaucoup de généraux, ou gouverneurs des colonies. Puis elle s'est de plus en plus composée de banquiers, hommes d'affaires, propriétaires, à quoi s'ajoute une proportion plus modeste d'universitaires, architectes, artistes, hommes politiques, même s'il est en principe interdit d'y parler politique.

Je rapproche volontiers cette organisation de celle de sociétés comme " Le Félibrige ", qui s'était associé dès sa création, en 1876, un groupement parisien des méridionaux de la capitale. On sait moins de l'histoire des « Bourguignons salés », qui serait à faire. Elle a sans doute aussi à voir avec la vague régionaliste de la fin du siècle dernier, manifestant, au-delà de la localisation parisienne des activités professionnelles de classes aisées issues des provinces, un souci de solidarité en référence aux origines territoriales et à la classe sociale, qui transcende les divergences personnelles en termes d'intérêts particuliers, de religion et d'opinions politiques.

Le géographe et Bourguignon Georges Chabot, professeur à l'Institut de géographie de Paris, lui ayant dans les années 60 proposé de lui succéder en qualité de membre actif, c'est à nouveau un hasard de la vie qui a fait Michel Rochefort membre de ce cénacle.

\section{NOTES}

1.ou Rétif: l'écriture n'est pas fixée, mais l'auteur (1734-1806) est connu à la fois comme le premier « écrivain paysan " peintre en particulier de la vie rurale de Sacy, et pour ses nombreux romans libertins situés à Paris où il a vécu l'essentiel de sa vie d'écrivainimprimeur.

2.Cette contribution est issue d'une journée d'entretien avec Michel Rochefort en août 1998 à Joux, de ses premiers articles, et de quelques observations personnelles sur le destin ultérieur des lieux de ses premiers travaux.

3.Ce bassin de Joux, Michel Rochefort s'évertue aujourd'hui encore à en expliciter la genèse, " bassin mio-pliocène ayant évolué sous climat tropical, avec reprise quaternaire et enfoncement du réseau hydrographique », comme si la genèse des formes du relief avait une importance spéciale pour l'intelligence du lieu. Il faudrait s'interroger de manière approfondie sur le souci de la génétique des formes, de toutes les formes des paysages de l'espace géographique qui a animé plusieurs générations de géographes. (Si j'en crois le Guide géologique de la Bourgogne, Paris, ed. Masson, la néo-tectonique y aurait également un rôle, mais je n'ai pas trouvé de travaux précis utilisant les acquis et méthodes de la géomorphologie des années 90 qui expliquent les nuances locales des reliefs des plateaux bourguignons).

4.Merger ou murger : amoncellements de pierres en bordure de champs, issus de l'épierrage des sols ( $C f$. photo infra).

5.Edme Restif de la Bretonne eut en effet 14 enfants de plusieurs mariages. $C f$. Nicolas Restif de la Bretonne, 1779, La vie de mon père.

6.0p. cit. 
7.Il faut se souvenir qu'il n'y avait alors ni assurances, ni retraites, et que les biens fonciers et immobiliers constituaient la réserve, en l'absence de valeurs mobilières dont on a vu comment la crise dite « de 1929 » les avait réduites à néant.

8.Le lac des Settons est au cœur du Morvan, à l'ouest d'Autun ; la forêt de Planoise est une vaste forêt domaniale, au sud-est, qui sépare Autun du Creusot.

9.Diplôme sur mémoire de recherches devenu « maîtrise » dans les années 70.

10.Michel Rochefort, 1952, « À propos des méthodes de la géographie agraire : le bocage récent d'Allerey ", Volume jubilaire du $\mathrm{L}^{\mathrm{e}}$ anniversaire du laboratoire de géographie de Rennes, Les nourritures terrestres, Rennes, p. 293-309.

11. Michel Rochefort, « Un exemple de l'influence des nouvelles techniques agricoles sur un budget paysan ", Bulletin de l'Association de géographes français $n^{\circ} 24-25$, mars-avril 1952, p. 70-74.

12.En 1949, le rendement n'avait pas sensiblement augmenté, puisque Michel Rochefort, dans « Un exemple de l'influence des nouvelles techniques agricoles sur un budget paysan ", op. cit., fait état de 14 q / ha. Mais l'extrême pauvreté semblait avoir disparu.

13.Nicolas Restif de la Bretonne, 1779, La vie de mon père.

14. Habitant de Sacy.

15.Certes les exemples sont choisis pour être comparables, mais l'on sait par ailleurs que la taille des fermes de carrière (on disait aussi « viables » à un moment donné) est restée en général assez stable durant cette période.

16.qui dominent le Conseil général de l'Yonne de manière permanente depuis la guerre.

17.Cf. supra note 9.

18. Michel Rochefort, 1949, La plaine d'Autun et ses bordures, étude de géographie agraire sous la direction de Jean Tricart, Strasbourg ; «La pénétration des capitaux bourgeois dans la campagne autunoise, ses conséquences sur l'habitat et la structure agraires », Revue de géographie de Lyon, tome 25, p. 249-266 (Cf. Réédition infra) ; Michel Rochefort, 1950, «L'évolution économique et sociale de Monthelon, Antully et Roussillon du Moyen-Âge au début du XIX ${ }^{\mathrm{e}}$ siècle », Mémoires de la Société éduenne, tome 50, 3e fascicule.

19.Cf. Pierre George, dir., 1959, Études de géographie rurale, supplément au Bulletin de Saint-Cloud, $208 \mathrm{p}$.

20.Thèse dont la généralité a été infirmée un peu plus tard, pour les plateaux nord du Bassin parisien, par Pierre Brunet qui montre l'avantage économique historiquement accumulé par les grands fermiers qui ne font qu'exploiter le foncier d'autrui sans en supporter la charge. Cf. Pierre Brunet, 1960, Structure agraire et économie rurale des plateaux tertiaires entre la Seine et l'Oise, Caen, Caron, 552 p.

21.Au sud-est d'Autun.

22.Issu d'observations et entretiens à Roussillon-en-Morvan et Monthelon réalisés par F. Plet en août 1998.

23. Groupement Agricole d'Exploitation en Commun, exploitation agricole impliquant le fonctionnement collectif de plusieurs chefs d'exploitation réunis en une même entité de production (loi de 1962).

24.Dits " prêts bonifiés », pour lesquels l'État paie aux banques la différence entre le taux d'intérêt courant et le taux préférentiel consenti aux agriculteurs.

25.Que le Parc Naturel Régional du Morvan remet actuellement en valeur.

26.Dans le courant des années 90 notamment. 
27.De 400 à 871 têtes selon les données du cahier de contrôle sanitaire durant cette période.

28.Issues de la PAC de 1992.

29. Coopératives d'Utilisation du Matériel Agricole.

30.Elles datent du début des années 80 , ce qui marque des conversions très tardives à l'achat et à l'utilisation collective de matériel si l'on se souvient que la politique favorisant les CUMA date de 1946.

31.Le marché italien est très important pour les éleveurs de bovins maigres (traduisez jeunes) car les grandes fermes d'engraissement de ce pays sont depuis près de trente ans les principales clientes des naisseurs français de races à viande.

32.Faiblesse des cours récurrente depuis 1985, le marché de la viande bovine ayant été successivement affecté par les quotas laitiers et les abattages massifs qui leur ont été associés, la maladie de la vache folle (ESB.) portée à la connaissance du public en 1989-90, puis, de manière plus inquiétante, en mars 1996 avec l'annonce de sa transmissibilité à l'homme.

\section{AUTEUR}

FRANÇOISE PLET

professeur à l'université de Paris 8-Saint Denis, Ladyss 